\title{
Transcriptional control of flavonoid biosynthesis in polyploid plant species
}

\author{
Strygina K.V. ${ }^{1,2 *}$, Mikhailova A.S. ${ }^{1,3}$, Khlestkina E.K. ${ }^{1,2}$ \\ ${ }^{1}$ N.I. Vavilov All-Russian Institute of Plant Genetic Resources, St. Petersburg, Russia \\ ${ }^{2}$ Institute of Cytology and Genetics, SB RAS, Novosibirsk, Russia \\ ${ }^{3}$ St. Petersburg State University, St. Petersburg, Russia \\ *e-mail:k.strygina@vir.nw.ru
}

Key words: flavonoids, MBW complex, cotton, wheat

Motivation and Aim: Gene duplication is the main evolution mechanism leading to the emergence of new gene functions and new species. Genomes of polyploid plants have an increased number of genes copies. Thereby, the studying of polyploid genomes is of interest to determine the functional and evolutionary features of the duplicated genes. Here, we report results of study on paralogous and homeologous copies of regulatory $M y b, b H L H$ and WD40 genes involved in flavonoid biosynthesis in monocot and dicot plant species - allotetraploid cotton Gossypium hirsutum L. $\left(\mathrm{AD}_{1}\right)$, allohexaploid bread wheat Triticum aestivum L. (BAD) and its relatives.

Methods and Algorithms: Homologous sequences of Myb, bHLH and WD40 genes were found in databases for not annotated wheat (Triticeae tribe) and cotton (Gossypium genus) sequences using BLAST search. The in silico analysis of identified sequences using Multalin, FGENESH+, New PLACE and InterPro software was carried out. The construction of the phylogenetic trees, the calculation of $K a / K s$ ratio and calculation of divergence time was made with MEGA software with 1000 bootstrap replicates.

Results: Using a wide range of bioinformatics tools, in Triticeae tribe we revealed three homologous clusters of $M y b$ genes, two clusters of $b H L H$ genes and two clusters of WD40 genes based on known sequences of TaCl (Myb), TaMycl (bHLH) and ZmPACl (WD40) genes. On the other hand, we identified tree paralogous clusters of Myb-coding genes, one group of $b H L H$ genes and two WD40 paralogous groups in Gossypium genus - orthologs of GhTT2, GhTT8 and AtTTG1, respectively. The results of investigation of phylogenetic relationship, divergence time and the analysis of structural organization of duplicated genes are represented. It was shown that members of WD40 gene family are mostly supported by selection than $M y b$ and $b H L H$. Additionally, the transcriptional activity of $M y b, b H L H$ and $W D 40$ genes in different parts of wheat plant was investigated. Conclusion: The results obtained in the current research revealed some genotype-specific features of phylogenetic relationship and structure organization of duplicated regulatory $M y b, b H L H$ and WD40 genes. In the future, the discovered genes could be used for the development of diagnostic DNA-markers in marker-assisted selection of cotton and wheat with naturally coloured tissues. 